\title{
Articulación de la influencia en Twitter ante el anuncio de la Ley del referéndum en Cataluña
}

\section{Constructing influence on Twitter facing the announcement of the Referendum Law in Catalonia}

\author{
Carlota M. Moragas-Fernández; Josep-Maria Grau-Masot; Arantxa Capdevila-Gómez
}

Cómo citar este artículo:

Moragas-Fernández, Carlota M.; Grau-Masot, Josep-Maria; Capdevila-Gómez, Arantxa (2019). “Articulación de la influencia en Twitter ante el anuncio de la Ley del referéndum en Cataluña". El profesional de la información, v. 28, n. 3, e280320.

https://doi.org/10.3145/epi.2019.may.20

Artículo recibido el 22-01-2018

Aceptación definitiva: 18-02-2019

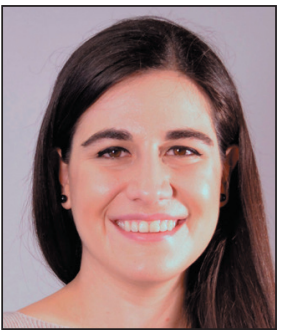

Carlota M. Moragas-Fernández https://orcid.org/0000-0003-3028-6663

Universitat Rovira i Virgili

Departamento de Estudios de Comunicación Av. Catalunya, 35. 43002 Tarragona, España carlotamaria.moragas@urv.cat $\bowtie$

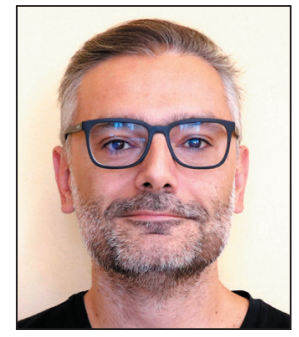

Josep-Maria Grau-Masot https://orcid.org/0000-0003-3484-2495

Universitat Rovira i Virgili

Departamento de Estudios de Comunicación Av. Catalunya, 35. 43002 Tarragona, España josepmaria.grau@urv.cat

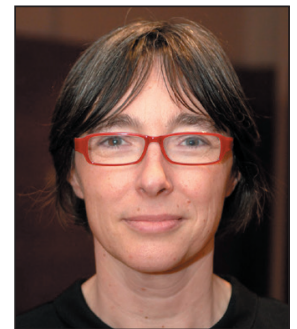

Arantxa Capdevila-Gómez

https://orcid.org/0000-0003-3030-0488

Universitat Rovira i Virgili

Departamento de Estudios de Comunicación

Av. Catalunya, 35. 43002 Tarragona, España

arantxa.capdevila@urv.cat

\section{Resumen}

En la línea de las investigaciones sobre los efectos de Twitter sobre la configuración de la opinión pública, se analizan las características del debate generado a raíz de la presentación de la Ley del referéndum de Cataluña. Se trabajó con dos muestras (n1: 260.866 tweets y retweets y n2: 1.681 tweets) que permitieron estudiar, por un lado, la morfología de la red de retweets (RT) y la obtención de los perfiles -líderes de influencia, constructores y puentes-que en ella se dibujan y, por otro, la conceptualización de la situación política por parte de aquellos usuarios que lideran la mencionada red. Los resultados muestran un porcentaje significativo (32\%) de usuarios de las esferas públicas periféricas entre los líderes de influencia, frente al $68 \%$ perteneciente a la esfera pública central. Ambos se configuran como líderes de dos comunidades altamente polarizadas ideológicamente a favor y en contra de esta Ley, lo cual se aleja del ideal de deliberación.

\section{Palabras clave}

Redes sociales; Medios sociales; Comunicación política; Esfera pública virtual; Twitter; Influencers; Metáfora; Polarización; Asuntos públicos; Análisis de redes sociales; Cataluña.

\section{Financiación}

Parte de esta investigación se ha llevado a cabo en el marco del proyecto de I+D "El papel de la metáfora en la definición y la percepción social de temas conflictivos. Instituciones, medios de comunicación y ciudadanos" [CSO201341661-P], financiado por el Ministerio de Economía y Competitividad.

Agradecimiento

Los autores agradecen al Dr. Roger Guimerà el apoyo en el diseño metodológico de esta investigación. 


\begin{abstract}
In line with the research carried out about the effects of Twitter on the configuration of public opinion, this article analyses the features of the debate generated as the result of the presentation of the Law of the Referendum of Catalonia. We work with two samples (n1: 260.866 tweets and retweets and n2: 1.681 tweets) in order to study, on the one hand, the morphology of the retweet (RT) network and the obtention of the profiles -influencers, builders and bridges- that articulate it and, on the other hand, the conceptualization of the political situation by the users who lead the aforementioned network. Results show that there is a significant percentage (32\%) of civil society users among influencers, compared to the $68 \%$ belonging to the central public sphere. Both profiles lead two ideologically polarized communities for and against this Law, moving away from the ideal of deliberation.
\end{abstract}

\title{
Keywords
}

Social networks; Social media; Political communication; Virtual public sphere; Twitter; Influencers; Metaphor; Polarization; Public issues; Social networks analysis; Catalonia.

\section{Introducción}

Antes de la aparición de la Web 2.0 la oportunidad de participación ciudadana en la opinión pública estaba esencialmente restringida al voto, aunque la esfera pública se concibiera como un espacio discursivo en el que los individuos actúan conjuntamente para asumir el rol de fuerza política poderosa (Ernst citado por McNair, 2007, p. 18). Para algunos autores (Sampedro, 2011; Barassi; Treré, 2012; Gil de Zúñiga; Molyneux; Zheng, 2014) este papel puede ejercerse de forma más plausible con la incorporación del entorno 2.0 y las tecnologías de la comunicación que se originan en él. En este sentido, Twitter, dado su carácter abierto, horizontal y en red (Sup-Park, 2013), concentra todos los atributos de la web 2.0-rapidez, naturaleza pública de la comunicación y posibilidad de interpelar a los usuarios (@mencionar), enlazar contenido externo y debatir alrededor de un tema (creando un hashtag o etiqueta) (Ausserhofer; Maireder, 2013:292)y se presenta como un instrumento que posibilita la deliberación entre políticos, medios y ciudadanos.

Twitter transforma la esfera pública tanto a nivel estructural como a nivel de influencia. En el plano estructural, la participación de la ciudadanía genera múltiples esferas públicas periféricas (EPP) que entran en interacción con la esfera pública central (EPC). Como afirma Sampedro (2000), a partir de Habermas:

- la esfera pública central tiene una estructura vertical, está dominada por las principales instituciones de la sociedad civil y genera debates cohesionados y formalizados sobre la gestión del poder y de los valores colectivos;

- las esferas públicas periféricas tienen una estructura horizontal, diversificada y líquida, están sostenidas por comunidades diversas que amplían la participación y contrarrestan a la esfera pública central.

En el plano de la influencia, el incremento de la participación de la ciudadanía y la diversificación de las comunidades presentes en la esfera pública ha dado lugar a dos visiones diferenciadas acerca del papel que desempeña Twitter en la articulación de esta cuestión.

Por un lado, algunos autores afirman que el entorno 2.0 refuerza la discusión democrática o la formación de una opinión pública deliberativa, al menos entre aquellos ciudadanos que actúan como público activo, (Margolis; Resnick, 2000; Dahlgren, 2005; López-García, 2006; Loader; Mercea, 2011; Sup-Park, 2013; Ausserhofer; Maireder, 2013; D’heer; Verdegem, 2014; Klinger; Svensson, 2015; Masip et al., 2015; Veenstra et al., 2015; Alvídrez; Franco-Rodríguez, 2016; Casero-Ripollés, 2018), aunque también señalen que la esfera pública virtual continúa reproduciendo las estructuras sociales existentes.

Por otro lado otros autores (McPherson; Smith-Lovin; Cook, 2001; Sustein, 2003; Yardi; Boyd, 2010; Feller et al., 2011; Gruzd, 2012; Aragón et al., 2013; Colleoni; Rozza; Arvidsson, 2014; Conover et al., 2011; Flaxman; Goel; Rao, 2016; Boutyline; Willer, 2017) consideran que, a través de las redes sociales -entre las que se incluye Twitter-, se refuerzan los propios puntos de vista, dada la exposición selectiva a los contenidos políticos a que están sometidos los usuarios. Estos autores se basan en la idea de que la diversidad de comunidades da lugar a un escenario fragmentado que, como afirman Colleoni, Rozza y Arvidsson (2014), opera mediante el mecanismo de la homofilia. McPherson, Smith-Lovin y Cook (2001) definen la homofilia como la propensión de las personas a agruparse según sus intereses y sus afinidades políticas. De este modo, la tendencia en Twitter es crear comunidades homogéneas que se convierten en cámaras de eco o de resonancia (Gruzd, 2012) dentro de las cuales los individuos sólo están expuestos a información y a comunidades que apoyan sus puntos de vista mientras que ignoran los puntos de vista opuestos, lo cual hace que las ideas se radicalicen (Yardi; Boyd, 2010; Sustein, 2008).

Sustein (2001), citado por Aragón et al. (2013, p. 186), afirma que en dichas cámaras de eco los usuarios sólo escuchan ecos más fuertes de sus propias voces y así reafirman sus propias opiniones.

Hsu y Park (2012); Boutet, Kim y Yoneki (2013) y Esteve-Del-Valle y Borge-Bravo (2018) han señalado que ocurre lo mismo en el nivel de los actores políticos. Esto se asocia con la adopción de posturas más extremas, que llevan a la polarización o incluso, como afirman Sustein (2003) y Aragón et al. (2013), a la balcanización de la interacción política en Twitter. 
Por otro lado, la investigación llevada a cabo por autores como Barberà et al. (2015), Vaccari et al. (2016), Barnidge (2017) o Dubois y Blank (2018) apunta a que las redes sociales son medios que incrementan el pluralismo político y la discusión entre distintas posturas ideológicas, por lo que las cámaras de eco serían la excepción y no la regla.

El objetivo de este trabajo es estudiar, a raíz de un suceso político conflictivo, las características del debate online y determinar si se puede hablar de una esfera pública que rompe con el modelo clásico de generación de la opinión pública mediante la existencia de múltiples comunidades y la interacción entre ellas. Para ello analizamos la actividad que generó en Twitter el anuncio, el 4 de julio de 2017, por parte del Govern de la Generalitat de Catalunya de la Ley del referéndum que daría lugar, meses más tarde, a la organización del mismo el 1 de octubre de 2017. Se trata de un suceso que forma parte del conflicto que desde 2012 enfrenta a la Generalitat y al Gobierno español (Guibernau, 2013; Serrano, 2013; Crameri, 2015) y en el que se discute sobre la posibilidad de convocar un referéndum de autodeterminación. Por lo tanto, se trata de un debate presente en la agenda pública del Estado, donde el posicionamiento de los actores que intervienen se negocia a través del discurso. Por ello este trabajo pretende:

- identificar si se trata de un debate polarizado o si existe cierta deliberación entre usuarios con diversos perfiles políticos;

- constatar cuál es la narrativa dominante sobre el hecho analizado.

\section{Articulación de la influencia en Twitter}

Al margen de la existencia o no de una esfera pública virtual cercana al ideal "habermassiano", son muchos los usuarios que gracias a los medios sociales participan en el debate político (Sup-Park, 2013; Alvídrez; Franco-Rodríguez, 2016). En este sentido, para Dahlgren (2005), el nuevo entorno comunicativo ha abierto el camino hacia la interacción cívica. Twitter se ha convertido en un espacio que potencia la proliferación de esferas públicas periféricas (Sampedro; Resina-De-la-Fuente, 2010) y que posibilita la apertura de un debate tradicionalmente restringido a la esfera pública central.

La tweetesfera se divide en clusters temáticos que forman parte de esferas más amplias dentro de las cuales aparecen asuntos de debate público que la organizan de una determinada manera. Grau-Masot (2018) habla de una esfera líquida con una estructura volátil que se organiza de modo diverso en cada asunto de debate público. Así, a nivel macroestructural, la infinidad de clústeres que conforman Twitter son líquidos, cambiantes, superpuestos entre sí desde el punto de vista de los perfiles, y poliédricos en lo que se refiere a los temas de interés. En consecuencia, en cada asunto de debate la red adopta una morfología diferente que se concreta en la aparición de comunidades ad hoc que se articulan a través de las herramientas comunicativas propias de Twitter: el retweet (RT) y la mención. Son precisamente estos instrumentos los que generan las redes de influencia (Gruzd, 2012; Conover et al., 2011). La aproximación a estas herramientas como mecanismos que permiten clasificar y definir los perfiles que participan en la conversación en Twitter se apoya en los trabajos previos de autores como Cha et al. (2010); Bakshy et al. (2011); Larsson y Moe (2011); Conover et al. (2011); Aragón et al. (2013); Dang-Xuan et al. (2013); González-Bailón, Borge-Holthoefer y Moreno (2013); Ausserhofer y Mareider (2013); Guerrero-Solé, Corominas-Murtra y López-González (2014); Jungherr y Jürgens (2014); Dubois y Gaffney (2014); Choi (2015); Yang; Quan-Haase; Rannenberg (2016); Feng (2016); Hilbert et al. (2016); Gonzalez-Bailón y Wang (2016); o Guerrero-Solé (2017).

Cha et al. (2010) compararon tres medidas de influencia (el número de seguidores y el número de RT y de menciones recibidas) y concluyeron que tener un número muy alto de seguidores no implica generar RT ni menciones, mientras que Bakshy et al. (2011) investigaron los atributos y la influencia relativa de 1,6 millones de usuarios haciendo el seguimiento de la difusión de 74 millones de eventos que se llevaron a cabo durante dos meses en 2009 y establecieron que la comunicación boca a boca -aquella buscada por las estrategias de marketing-funciona de forma más eficaz con los líderes de influencia "corrientes", es decir, aquellos que ejercen una influencia alrededor o por debajo de la media.

Larsson y Moe (2011) aplicaron el análisis de redes sociales al debate sobre las elecciones suecas de 2010 e identificaron distintos perfiles de usuarios de la red de RT (Retweeters, Elites, Networkers) y de menciones (Senders, Receivers, Sender-receivers).

Dang-Xuan et al. (2013) obtuvieron los perfiles más influyentes a partir del número de RT recibidos y llevaron a cabo un análisis de contenido de sus tweets y Dubois y Gaffney (2014) hicieron lo propio comparando seis métricas de análisis de redes sociales.

González-Bailón, Borge-Holthoefer y Moreno (2013) plantearon cuatro tipos de usuarios en función de su rol (Influentials, Hidden influentials, Broadcasters y Common users) durante el crecimiento de las movilizaciones en España en 2011. Del mismo modo, Jungherr y Jürgens (2014) determinaron los perfiles más influyentes a partir de los RT y menciones recibidas durante una campaña de protesta en Alemania.

Ausserhofer y Mareider (2013) argumentaron que Twitter permitía el acceso a nuevos actores a la esfera política tradicional, midiendo la influencia a partir del número de seguidores y de las menciones.

Choi (2015) determinó que el modelo Two-step flow aún tenía validez y que los líderes de opinión eran influyentes, pero no creadores de contenido. Así lo hicieron Hilbert et al. (2016), quienes apuntaron que dicho modelo convivía con otros tipos de flujos como el directo One-step y otros más complejos. 
Yang et al. (2016) y Feng (2016) obtuvieron los perfiles más influyentes a partir del seguimiento de debates generados en Twitter vía hashtags. Además, Feng (2016) planteó cinco tipos de usuarios centrales: Conversation starter, Influencer, Active engager, Network builder e Information bridge. Precisamente González-Bailón y Wang (2016) analizaron la red de comunicación surgida en el contexto de una campaña de protesta en 2012 y determinaron los perfiles potenciales que creaban puentes para la difusión de la información, y Guerrero-Solé (2017) desarrolló un método de análisis (RON) que considera que las superposiciones entre las comunidades de los retweeters podrían usarse como un predictor de la percepción que los votantes tienen en relación con la distancia ideológica entre usuarios.

Conover et al. (2011) demuestran que la red de RT exhibe un alto grado de estructura modular y segrega a los usuarios en comunidades homogéneas mientras que en la red de menciones no se produce este fenómeno. A esta misma conclusión llegan Aragón et al. (2013) para el caso de la tweetesfera española en la que se detecta un escaso intercambio de RT entre miembros de diferentes partidos políticos, es decir, sólo se comparte información generada en la propia red. Sin embargo, la polarización de la red no está reñida con la diversidad de la tweeteresfera. En ella los perfiles de la esfera pública central, como políticos y periodistas, ejercen la influencia junto a otros perfiles ciudadanos y forman comunidades específicas para cada asunto tratado. Ya no se trata del liderazgo de opinión fundamentado en el "two-step flow communication" descrito por Katz y Lazarsfeld (1955), que se sostiene en un modelo comunicativo vertical en el que el ciudadano es considerado auditorio pasivo. Hoy en día es razonable afirmar que este esquema está como mínimo incompleto.

De este modo, si el hecho digital e internet han provocado la reformulación de la esfera pública a nivel estructural, es lógico pensar que los actores que influyen en la creación de opinión pública también se hayan visto alterados y que, en consecuencia, en la tweetesfera exista un mayor grado de variabilidad en el flujo de influencia. En ella se dan diversos flujos que configuran un espacio donde el concepto de influencia se amplía más allá del líder de opinión (Dang-Xuan et al., 2013).

Un elemento clave en la articulación de la influencia en Twitter es, como se ha apuntado sobre estas líneas, el RT (González-Bailón; Wang, 2016). Entendemos el RT como la práctica de reenviar un tweet de otro usuario (Larsson; Moe, 2011). Ello conlleva que el tweet en cuestión tiene destinatarios adicionales además de los seguidores del autor del tweet. Es precisamente la capacidad de difusión del RT lo que convierte a Twitter en una plataforma ideal desde el punto de vista del usuario para diseminar informaciones y opiniones (Dang-Xuan et al., 2013, p. 2) y por ello, este trabajo se centra en su estudio. El hecho de que un tweet tenga una gran cantidad de RT en comparación con el número total de RT producidos dentro de una red, dota de influencia a su autor dado que tiene más audiencia (Cha et al., 2010). En el contexto de una red de RT generada alrededor de un tema de debate público (Bruns; Highfield, 2015) conviven distintos perfiles cuyo análisis permite definir la articulación de la influencia en Twitter. En dicha red se genera un modelo de influencia distribuida en la que cada usuario es un nodo y la influencia recae en los perfiles que posibilitan la construcción de la esfera pública, esto es:

- los líderes de influencia (influencers): son los perfiles más retweeteados;

- los constructores: son los perfiles que retweetean más;

- los puentes: son los perfiles que retweetean más y a la vez son más retweeteados.

Así, en la discusión de un tema público, los líderes de influencia son los perfiles que reciben el mayor apoyo de la red formada, y quienes potencialmente, mediante el contenido de sus tweets, pueden ser creadores de opinión pública. Los constructores son los perfiles más activos de la red en el sentido de difusión de contenido a sus respectivos auditorios. $Y$ los puentes son los perfiles que potencialmente pueden gestionar el mayor flujo de información de la red.

Si bien es cierto que la morfología de la red de RT brinda una fotografía de los actores que interactúan en la esfera pública virtual y apunta quienes ejercen como líderes en la conversación, es necesario evaluar cualitativamente el contenido de la misma si queremos determinar hasta qué punto cuestiones como la polarización entre comunidades de usuarios se reproduce en el plano discursivo. Esto es especialmente relevante en el caso de los líderes de influencia que se erigen en los perfiles cuyos tweets tienen mayor difusión. Las metáforas, como mecanismos lingüísticamente económicos -y por ello, con mayor probabilidad de ser usados estratégicamente en afirmaciones de 140 caracteres $^{1}$ - con un evidente componente persuasivo, cognitivo e ideológico en su uso en el discurso público (Musolff, 2004; 2006; 2012; Charteris-Black, 2011; Semino, 2008), permiten identificar los posicionamientos discursivos y las estrategias de comunicación que en ellos subyacen (Moragas-Fernández; Montagut; Capdevila, 2018). Más allá de la concepción clásica de las expresiones metafóricas como frame devices (Gamson; Modigliani, 1989), con frecuencia éstas también constituyen marcos de interpretación figurativos en sí mismas (Burgers; Konijn; Steen, 2016). En este sentido, su uso en el discurso de los líderes de influencia en Twitter se presenta como una forma concreta de aproximación al conflicto político analizado y a su discusión entre comunidades.
Los influencers son los perfiles más retweeteados, los constructores son los que retweetean más y los puentes son los que retweetean más y a la vez son más retweeteados 


\section{Metodología}

La presente investigación trabaja con dos muestras $(\mathrm{n} 1, \mathrm{n} 2)$ que permiten:

(O1) Estudiar la red de RT, con el objetivo de determinar cómo se formaron las comunidades de usuarios que deliberaron en torno al anuncio de la Ley de Referéndum, si hubo comunicación entre dichas comunidades y quiénes se erigieron en líderes de influencia en ellas.

(O2) A partir de la detección de estos líderes o generadores de opinión, aproximarnos cualitativamente a la forma en que dichos actores conceptualizan la situación y la trasladan a los demás participantes en la conversación.

\subsection{Obtención de las muestras (n1, n2)}

La muestra (n1) está integrada por un total de 260.866 tweets y RT únicos emitidos entre los días 4 y 5 de julio de 2017, que se obtienen a partir de una búsqueda mediante palabras clave y hashtags (HT) relacionados con el caso de estudio y tras haber filtrado los tweets y RT repetidos, como consecuencia de las distintas búsquedas realizadas. Como puede verse en la tabla 1, con el fin de conseguir una fotografía lo más amplia y veraz posible a pesar de las restricciones de acceso a la información de la API de Twitter (Witschge et al., 2016), se consideró que la búsqueda basada únicamente en HT habría conllevado importantes limitaciones, dado que éstos no siempre son usados por los usuarios que participan en la discusión (Larsson; Moe, 2011). El código empleado para la obtención de los tweets y para la preparación de los datos se ha elaborado con el lenguaje estadístico $R$, mediante el cual nos comunicamos con la API de Twitter con la modalidad REST API (Search Twitter). El análisis de los datos, la cuantificación de las métricas y la representación gráfica de las redes se ha llevado a cabo con el software libre Gephi 0.9.2.

La muestra (n2) está compuesta por 1.681 tweets emitidos entre los días 4 y 5 de julio de 2017 por aquellos perfiles que, a partir del cálculo de diferentes métricas de análisis de redes sociales, se han determinado como influyentes. La identificación de estos perfiles permite conocer no sólo su estrategia discursiva sino también su procedencia y facilita el hecho de determinar si, en el caso del estudio analizado, Twitter rompe con el concepto clásico de esfera pública, permitiendo que nuevos actores pertenecientes a las esferas públicas periféricas adquieran relevancia o influencia en la construcción de la opinión pública en el entorno 2.0 .

\subsection{Metodología de análisis y operacionalización de los datos}

\subsubsection{Líderes de influencia, constructores y puentes en la red de RT}

El análisis de la muestra (n1) se ha llevado a cabo mediante una clasificación de perfiles directamente vinculada al "Degree”, que es una métrica cuantitativa englobada dentro del concepto de centralidad en el análisis de redes sociales (Scott; Carrington, 2012; Scott, 2013; Cherven, 2015). La centralidad permite identificar los nodos más relevantes de una red y asociar funciones comunicativas a los perfiles identificados en el marco de la red de influencia. Así, primero se identifica a los líderes de influencia, a los constructores y a los puentes de la red de RT y, después, se les clasifica en comunidades según la esfera pública a la que pertenecen y el posicionamiento a favor o en contra de la independencia de Cataluña o de sus líderes.

Para identificar estos perfiles se han usado diferentes métricas de análisis de redes sociales. Se ha determinado que los líderes de influencia son los perfiles que reciben el mayor número de enlaces (RT) ponderados (Weighted Indegree - WI), y que obtienen el $50 \%$ del total de enlaces de la red formada a raíz del asunto tratado. Además se ha tenido en cuenta la relevancia de esos enlaces mediante la Authority Centrality ( $\mathrm{AC}$ ), métrica que nos permite establecer en qué medida un nodo es relevante en función de los enlaces de salida que generan sus nodos vecinos. Así, un nodo será relevante si sus nodos vecinos apuntan a otros nodos también relevantes (Newman, 2010). La posible desviación de esta métrica respecto a la WI proporciona indicios de la posible polarización entre comunidades, en el caso de que estas tuvieran diferencias de impacto dentro de la red global.
Tabla 1. Número de tweets por hashtag $(\mathrm{HT})$ y palabras clave empleadas para la configuración de la muestra (n1)

\begin{tabular}{|c|c|c|c|c|c|}
\hline & HT & $\begin{array}{l}\text { Palabra } \\
\text { clave }\end{array}$ & Día 4 & Día 5 & Total \\
\hline Catalonia & & $x$ & 10.917 & 6.223 & 17.140 \\
\hline Cataluña & & $x$ & 15.091 & 19.388 & 34.479 \\
\hline Catalunya & & $x$ & 22.134 & 26.728 & 48.862 \\
\hline Referendum & & $x$ & 77.815 & 68.106 & 145.921 \\
\hline $10 C \mathrm{~T}$ & $x$ & & 945 & 1.041 & 1.986 \\
\hline $10 C T 2017$ & $x$ & & 1.023 & 355 & 1.378 \\
\hline 10ctubre & $x$ & & 143 & 163 & 306 \\
\hline 1Octubre2017 & $x$ & & 50 & 67 & 117 \\
\hline Catalonia & $x$ & & 3.579 & 1.200 & 4.779 \\
\hline Cataluña & $x$ & & 394 & 606 & 1.000 \\
\hline Catalunya & $x$ & & 795 & 926 & 1.721 \\
\hline ComSempre & $x$ & & 10.147 & 7.640 & 17.787 \\
\hline Independència & $x$ & & 1.166 & 3.839 & 5.005 \\
\hline LleiReferendum & $x$ & & 331 & 144 & 475 \\
\hline Referendum & $x$ & & 5.554 & 4.313 & 9.867 \\
\hline ReferendumCAT & $x$ & & 3.609 & 1.978 & 5.587 \\
\hline
\end{tabular}


Por otro lado se consideran constructores aquellos perfiles que generan el mayor número de RT ponderados (Weighted Outdegree - WO), y que generan el 50\% del total de enlaces existentes en la red. Una vez detectados se calcula su Hub Centrality para justificar las posibles desviaciones de AC de algunos líderes de influencia, sobre todo la de aquellos constructores que pertenecen a sus mismas comunidades. Esta métrica determina la relevancia de un nodo a partir de los enlaces que genera y de la importancia de dichos enlaces (Newman, 2010).

Por último, los perfiles que actúan como puentes se definen como aquellos que reciben un mayor número de enlaces y al mismo tiempo, los generan. Para ello se han filtrado de manera cruzada la WI y la WO y se han fijado valores de umbral mínimos en ambas medidas con la finalidad de establecer de forma concreta y proporcional qué usuarios enlazan comunidades en la red analizada.

\subsubsection{Una aproximación cualitativa a los líderes de influencia}

Por otro lado, para analizar la muestra (n2) se ha empleado una metodología cualitativa fundamentada en el Critical Metaphor Analysis (CMA) propuesto por Charteris-Black (2004). El CMA se concreta en tres estadios:

- identificación de la metáfora;

- interpretación de la metáfora;

- explicación de la metáfora.

Analíticamente, en la primera fase se detectan las palabras que remiten a campos semánticos diferentes a aquel sobre el que se está hablando. Se trata de la tensión semántica (Charteris-Black, 2004) o de la ruptura de la isotopía (Greimas, 1987). Estas palabras, que remiten a conceptos diversos al tema tratado, pueden ser interpretadas como señales o token (Kövecses, 2010) que indican la presencia de metáforas y se agrupan siguiendo criterios de similitud semántica que posibilitan su clasificación dentro de unidades conceptuales más amplias (source domains). La interpretación y la explicación se llevan a cabo relacionando los aspectos que se destacan del source domain identificado (p. ej. viaje/camino/ movimiento) con el target domain (p.ej. Ley del referéndum). Dicha relación genera mappings o conexiones sistemáticas entre elementos constitutivos de los dos dominios conceptuales y nos permite determinar la metáfora (p. ej. "La Ley del referéndum encarrila el ejercicio democrático del derecho a voto" Benet Salellas vía @DiariARA). Por último, la introducción del contexto nos permite entender qué narrativa o definición de la situación se deriva del uso de las metáforas en cuestión.

La codificación de la muestra se ha llevado a cabo de forma manual por dos investigadoras de acuerdo con los siguientes parámetros:

Tabla 2. Elementos de codificación de la muestra (n2)

\begin{tabular}{|c|c|c|c|c|c|c|c|}
\hline Fecha & Actor & Tweet & Hashtag & Metáfora & $\begin{array}{l}\text { Source } \\
\text { domain }\end{array}$ & $\begin{array}{l}\text { Target } \\
\text { domain }\end{array}$ & Comentario \\
\hline $4 / 7 / 17$ & $\begin{array}{l}\text { Gabriel Rufián } \\
\text { @gabrielrufian }\end{array}$ & $\begin{array}{l}\text { Yo soy de un país en el que se } \\
\text { explica como votar desde un } \\
\text { Parlament y no como conspirar } \\
\text { desde un Ministerio. }\end{array}$ & No hay & $\begin{array}{l}\text { conspirar } \\
\text { desde un } \\
\text { Ministerio }\end{array}$ & $\begin{array}{l}\text { Conflicto/ } \\
\text { guerra }\end{array}$ & $\begin{array}{l}\text { Gobierno de } \\
\text { España }\end{array}$ & \\
\hline
\end{tabular}

La concordancia entre las codificaciones de los investigadores se ha calculado aplicando la fórmula sugerida por Holsti (1969): C.R. = 2M/N1 + N2, donde (M) es el número de codificaciones en que estos coinciden y N1 y N2 las decisiones tomadas por cada investigador. Aplicando esta fórmula a un $25 \%$ de la muestra se han obtenido unos índices de 0,86 en la identificación de las metáforas, 0,89 en la del source domain y 0,84 en el target domain, todos ellos niveles aceptables $(0,75-1,0)$.

\section{Resultados y discusión}

La red global de RT del caso estudiado está conformada por 41.688 nodos y 124.787 enlaces ponderados (155.396 enlaces reales), cuya representación gráfica puede apreciarse en la figura 1. En ella se visualizan, diferenciadas por color, las principales comunidades (12) que conforman la red $(95,4 \% \text { de los nodos) })^{2}$, el porcentaje de habitantes de cada una de ellas, así como los nombres de los perfiles que las lideran con el mayor valor de WI. Visualmente podemos apreciar que la densidad de líderes de influencia de las comunidades de la parte izquierda de la red es superior a la de la derecha.

Las comunidades que dominan la parte derecha son la 16 (color verde claro) y la 17 (color naranja claro). La primera, está liderada por el eurodiputado @jordi_canyas, la asociación Convivencia Cívica (@CCivicaCatalana), el perfil anónimo @Er_Richal, el diario digital @okdiario y la asociación Societat Civil Catalana (@Societatcc).

La comunidad 17 está liderada por la agencia de noticias @europapress, el partido político @PSOE, el diario digital @eldiarioes, el diario @el_pais y el secretario general del PSOE @sanchezcastejon.

Prácticamente todos los perfiles que lideran ambas comunidades se posicionan en contra de la Ley del referéndum y representan un $31,12 \%$ de la población de la red y un $21,02 \%$ de los enlaces. Si se considera la red de RT como una red de apoyo, podemos afirmar que los nodos que habitan en estas comunidades mayoritariamente apoyan a estos perfiles y, en consecuencia, también estarían en contra de la Ley del referéndum. Estos datos nos permiten catalogar a las comunidades 16 y 17 como contrarias al referéndum. 
Comunidad $16 \mid$ En contra del referéndum - $(23,49 \%)$

Comunidad 17 | En contra del referéndum - (7,63\%)

Comunidad $18 \mid \mathrm{A}$ favor de un referéndum pactado - (1,36\%)

- Comunidad $6 \mid$ A favor del referéndum - $(19,82 \%)$

Comunidad 24 | A favor del referéndum - (14,64\%)

Comunidad 1 | A favor del referéndum - $(6,74 \%)$

Comunidad $23 \mid \mathrm{A}$ favor del referéndum - $(4,01 \%)$

- Comunidad $25 \mid \mathrm{A}$ favor del referéndum - $(2,99 \%)$

Comunidad 2 | A favor del referéndum - $(2,37 \%)$

Comunidad $0 \mid \mathrm{A}$ favor del referéndum - $(1,96 \%)$

- Comunidad $13 \mid \mathrm{A}$ favor del referéndum - $(4,97 \%)$

Comunidad $15 \mid$ A favor del referéndum - $(5,43 \%)$

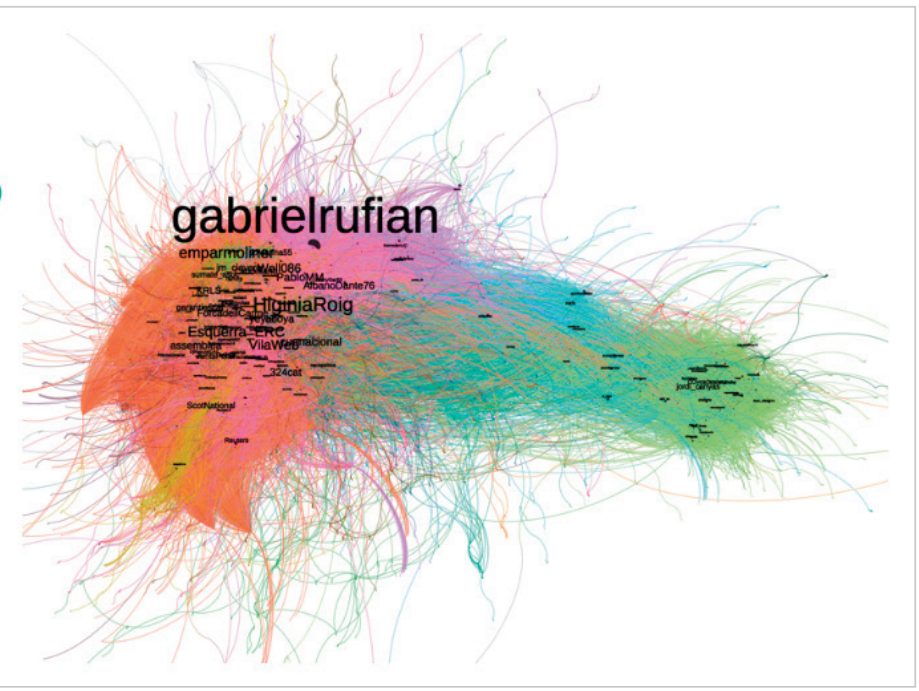

Figura 1. Principales comunidades que conforman la red global de RT del caso de estudio

La comunidad 18 (azul celeste), con un 1,36\% de los nodos y un 0,66\% de los enlaces, está liderada por perfiles que defienden un referéndum pactado con el Estado, aunque con presencia de ciertos perfiles independentistas, como lo demuestra el hecho que esté liderada por la agrupación de independentistas de CCOO, @indepesCCOO, además de CCOO Cataluña, @ccoocatalunya, su secretario general @PachecoJpacheco, el partido político @EnComu_Podem y la Teniente Alcalde de Barcelona en Comú, @janetsanz.

Las principales comunidades con nodos mayoritariamente favorables al referéndum se encuentran situadas en la zona izquierda de la imagen y suman el $62,92 \%$ de los nodos y el 73,74 de los enlaces. Se trata de comunidades como por ejemplo la 6 (color fucsia), cuyos perfiles más activos son el diputado del Congreso por ERC @gabrielrufian, el periodista anónimo @PabloMM, el entonces diputado de la Generalitat por CSQP @AlbanoDante76, el activista anónimo @JuanyGar63 y el exdirigente de Podemos @MonederoJC; o la 24 (color naranja) donde encontramos perfiles como el periódico digital @Vilaweb, activistas anónimos como @Well086o@Candeliano, la asociación @sumate_asocy su presidente @jm_clavero.

Las comunidades 1 (color violeta), 23 (color rosa), 25 (color amarillo oscuro), 2 (color salmón), 0 (color azul claro) y 13 (color azul oscuro) también están habitadas por perfiles que apoyan el referéndum, aunque son comunidades con un menor número de nodos y enlaces.

De acuerdo con la morfología que presenta la red de RT en el caso del anuncio de la Ley del referéndum, podemos afirmar que el peso de la conversación lo llevan las comunidades cuyos líderes se muestran claramente favorables a lo que regula esta Ley: la celebración de un referéndum de autodeterminación.

La tabla 3 detalla los 87 líderes de influencia de la red de RT (se muestran gráficamente en la figura 2), ordenados según la comunidad a la que pertenecen, y según su valor de AC. Los nodos que se muestran en color gris tienen un valor muy bajo en esta métrica y pertenecen a las comunidades 16 y 17 , situadas en la parte derecha de la red general (figura 1)

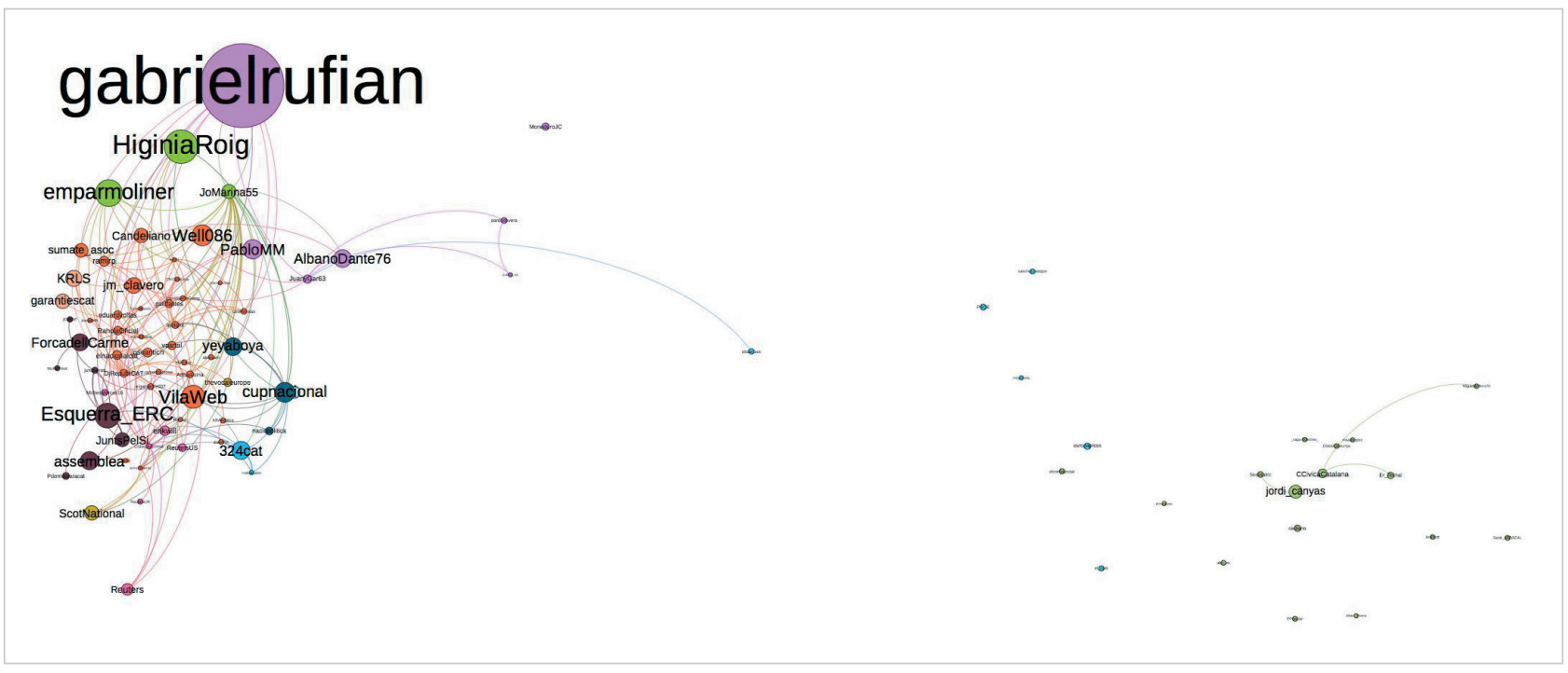

Figura 2. Mapa de líderes de influencia en la red de RT 
Tabla 3. Identificación de líderes de influencia en la red de RT

\begin{tabular}{|c|c|c|c|c|c|c|c|}
\hline & Perfil & Indegree & Authority & $\begin{array}{l}\text { Ranking } \\
\text { authority }\end{array}$ & Comunidad & $\begin{array}{l}\text { Pertenencia } \\
\text { EPC/EPP }\end{array}$ & Clasificación \\
\hline 1 & gabrielrufian & 7.559 & 0,59 & 1 & 6 & EPC & Actor político \\
\hline 2 & HiginiaRoig & 3.034 & 0,19 & 4 & 15 & EPC & Actor mediático \\
\hline 3 & emparmoliner & 2.417 & 0,25 & 2 & 15 & EPC & Actor mediático \\
\hline 4 & Esquerra_ERC & 2.238 & 0,11 & 16 & 1 & EPC & Actor político \\
\hline 5 & VilaWeb & 2.083 & 0,15 & 8 & 24 & EPC & Actor mediático \\
\hline 6 & Well086 & 1.887 & 0,19 & 3 & 24 & EPP & Activista Anónimo \\
\hline 7 & cupnacional & 1.790 & 0,08 & 25 & 13 & EPC & Actor político \\
\hline 8 & PabloMM & 1.743 & 0,15 & 7 & 6 & EPP & Activista Anónimo \\
\hline 9 & assemblea & 1.639 & 0,10 & 18 & 1 & EPC & Actor político \\
\hline 10 & 324 cat & 1.629 & 0,10 & 17 & 0 & EPC & Actor mediático \\
\hline 11 & yeyaboya & 1.612 & 0,16 & 5 & 13 & EPC & Actor político \\
\hline 12 & AlbanoDante76 & 1.598 & 0,14 & 11 & 6 & EPC & Actor político \\
\hline 13 & ForcadellCarme & 1.545 & 0,13 & 14 & 1 & EPC & Actor político \\
\hline 14 & KRLS & 1.454 & 0,14 & 12 & 2 & EPC & Actor político \\
\hline 15 & jm_clavero & 1.426 & 0,16 & 6 & 24 & EPC & Actor político \\
\hline 16 & garantiescat & 1.325 & 0,06 & 38 & 2 & EPC & Actor político \\
\hline 17 & ScotNational & 1.312 & 0,05 & 51 & 25 & EPC & Actor mediático \\
\hline 18 & Candeliano & 1.299 & 0,15 & 9 & 24 & EPP & Activista Anónimo \\
\hline 19 & JuntsPelSi & 1.267 & 0,08 & 27 & 1 & EPC & Actor político \\
\hline 20 & JoMarina55 & 1.266 & 0,13 & 13 & 15 & EPP & Ciudadano activista \\
\hline 21 & sumate_asoc & 1.243 & 0,15 & 10 & 24 & EPC & Actor político \\
\hline 22 & jordi_canyas & 1.189 & 0,0004 & 1.487 & 16 & EPC & Actor político \\
\hline 23 & Reuters & 1.059 & 0,03 & 87 & 23 & EPC & Actor mediático \\
\hline 24 & ramirp & 903 & 0,12 & 15 & 24 & EPP & Ciudadano activista \\
\hline 25 & erikwill & 883 & 0,07 & 28 & 23 & EPP & Ciudadano activista \\
\hline 26 & joseantich & 820 & 0,10 & 19 & 24 & EPC & Actor mediático \\
\hline 27 & elnacionalcat & 819 & 0,08 & 23 & 24 & EPC & Actor mediático \\
\hline 28 & CCivicaCatalana & 792 & 0,0002 & 2.008 & 16 & EPP & Plataforma activista \\
\hline 29 & RaholaOficial & 779 & 0,08 & 24 & 24 & EPC & Actor mediático \\
\hline 30 & eduardvoltas & 762 & 0,09 & 20 & 24 & EPC & Actor mediático \\
\hline 31 & vpartal & 759 & 0,08 & 26 & 24 & EPC & Actor mediático \\
\hline 32 & JuanyGar63 & 754 & 0,04 & 67 & 6 & EPP & Ciudadano activista \\
\hline 33 & thevocaleurope & 751 & 0,04 & 65 & 25 & EPC & Actor mediático \\
\hline 34 & ReutersUS & 726 & 0,04 & 61 & 23 & EPC & Actor mediático \\
\hline 35 & gallifantes & 711 & 0,08 & 22 & 24 & EPP & Ciudadano activista \\
\hline 36 & naciopolitica & 706 & 0,05 & 47 & 13 & EPC & Actor mediático \\
\hline 37 & DjRepubliCAT & 700 & 0,07 & 31 & 24 & EPP & Activista Anónimo \\
\hline 38 & leotaxil & 650 & 0,07 & 32 & 24 & EPP & Activista Anónimo \\
\hline 39 & MonederoJC & 646 & 0,02 & 127 & 6 & EPC & Actor político \\
\hline 40 & Pdemocratacat & 626 & 0,04 & 75 & 1 & EPC & Actor político \\
\hline 41 & AdriaAlsina & 617 & 0,09 & 21 & 24 & EPP & Ciudadano activista \\
\hline 42 & Er_Richal & 583 & 0,0001 & 2.254 & 16 & EPP & Activista Anónimo \\
\hline
\end{tabular}




\begin{tabular}{|c|c|c|c|c|c|c|c|}
\hline 43 & okdiario & 579 & 0,0007 & 1.145 & 16 & EPC & Actor mediático \\
\hline 44 & europapress & 578 & 0,0025 & 537 & 17 & EPC & Actor mediático \\
\hline 45 & MoltesMerces16 & 568 & 0,04 & 53 & 23 & EPP & Parodia \\
\hline 46 & junqueras & 558 & 0,07 & 30 & 1 & EPC & Actor político \\
\hline 47 & elgatnegre007 & 539 & 0,04 & 58 & 24 & EPP & Activista Anónimo \\
\hline 48 & PSOE & 537 & 0,0009 & 1.010 & 17 & EPC & Actor político \\
\hline 49 & pardodevera & 531 & 0,03 & 98 & 6 & EPC & Actor mediático \\
\hline 50 & Societatcc & 530 & 0,0001 & 2.248 & 16 & EPC & Actor político \\
\hline 51 & ceskfreixas & 519 & 0,07 & 33 & 24 & EPP & Ciudadano activista \\
\hline 52 & rfernsan & 497 & 0,05 & 48 & 24 & EPP & Ciudadano activista \\
\hline 53 & CollectiuEmma & 493 & 0,06 & 62 & 23 & EPP & Plataforma activista \\
\hline 54 & jcuixart & 493 & 0,04 & 36 & 1 & EPC & Actor político \\
\hline 55 & eldiarioes & 487 & 0,01 & 308 & 17 & EPC & Actor mediático \\
\hline 56 & EspanyaEnComu & 486 & 0,06 & 35 & 24 & EPP & Parodia \\
\hline 57 & ramontremosa & 486 & 0,05 & 43 & 24 & EPC & Actor político \\
\hline 58 & ARApolitica & 483 & 0,05 & 44 & 24 & EPC & Actor mediático \\
\hline 59 & aleixsarri & 478 & 0,06 & 41 & 24 & EPC & Actor político \\
\hline 60 & elconfidencial & 478 & 0,004 & 384 & 16 & EPC & Actor mediático \\
\hline 61 & el_pais & 470 & 0,001 & 806 & 17 & EPC & Actor mediático \\
\hline 62 & ReutersUK & 463 & 0,03 & 85 & 23 & EPC & Actor mediático \\
\hline 63 & raulromeva & 460 & 0,05 & 45 & 1 & EPC & Actor político \\
\hline 64 & Santi_ABASCAL & 448 & $2,60 \mathrm{E}-005$ & 3.163 & 16 & EPC & Actor político \\
\hline 65 & PPopular & 447 & $3,88 \mathrm{E}-004$ & 1.604 & 16 & EPC & Actor político \\
\hline 66 & sanchezcastejon & 442 & $4,59 \mathrm{E}-004$ & 1.462 & 17 & EPC & Actor político \\
\hline 67 & abc_es & 437 & 0,001 & 782 & 16 & EPC & Actor mediático \\
\hline 68 & diariARA & 436 & 0,05 & 52 & 24 & EPC & Actor mediático \\
\hline 69 & DolcaCatalunya & 434 & $1,35 \mathrm{E}-004$ & 2.283 & 16 & EPP & Plataforma activista \\
\hline 70 & CNICatalunya & 422 & 0,06 & 40 & 24 & EPP & Parodia \\
\hline 71 & eduardcabus & 418 & 0,07 & 34 & 24 & EPP & Ciudadano activista \\
\hline 72 & jcanadellb & 418 & 0,06 & 29 & 24 & EPP & Ciudadano activista \\
\hline 73 & JosPastr & 417 & $1,14 \mathrm{E}-004$ & 2.388 & 16 & EPP & Activista Anónimo \\
\hline 74 & MigueldelAmo70 & 415 & $4,71 \mathrm{E}-005$ & 2.964 & 16 & EPP & Ciudadano activista \\
\hline 75 & maticatradio & 401 & 0,02 & 109 & 0 & EPC & Actor mediático \\
\hline 76 & MonDiari & 399 & 0,04 & 55 & 24 & EPC & Actor mediático \\
\hline 77 & _cagandoleche_ & 396 & $8,06 \mathrm{E}-005$ & 2.605 & 16 & EPP & Parodia \\
\hline 78 & rosadiezglez & 394 & $1,59 \mathrm{E}-004$ & 2.187 & 16 & EPC & Actor político \\
\hline 79 & CarlesMundo & 393 & 0,06 & 37 & 24 & EPC & Actor político \\
\hline 80 & directe & 391 & 0,04 & 60 & 24 & EPC & Actor mediático \\
\hline 81 & elmundoes & 386 & $7,78 \mathrm{E}-004$ & 1.106 & 16 & EPC & Actor mediático \\
\hline 82 & Albert_Rivera & 385 & $1,80 \mathrm{E}-004$ & 2.100 & 16 & EPC & Actor político \\
\hline 83 & miqueliceta & 383 & $8,25 \mathrm{E}-004$ & 1.078 & 17 & EPC & Actor político \\
\hline 84 & egel71 & 377 & 0,06 & 39 & 24 & EPP & Ciudadano activista \\
\hline 85 & publico_es & 370 & 0,02 & 118 & 6 & EPC & Actor mediático \\
\hline 86 & JanneRiitakorpi & 369 & 0,03 & 92 & 24 & EPP & Ciudadano activista \\
\hline 87 & pilarcarracelas & 367 & 0,06 & 42 & 24 & EPC & Actor mediático \\
\hline
\end{tabular}




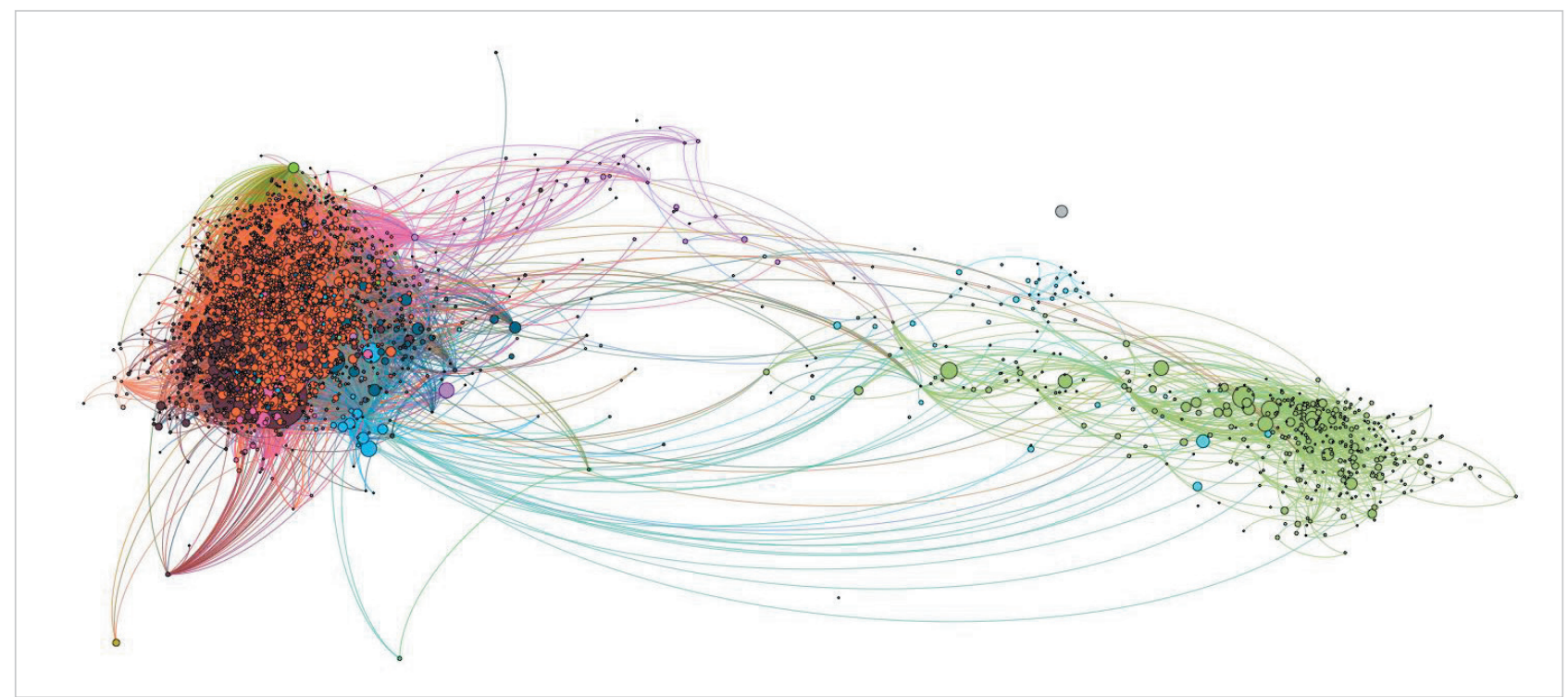

Figura 3. Mapa de constructores en la red de RT

y lideradas por perfiles posicionados mayoritariamente en contra del referéndum. Esto implica que sus comunidades tienen un menor impacto en la red global.

La delimitación de los líderes de influencia y su clasificación de acuerdo con su pertenencia a la EPC o la EPP nos permite apuntar que existe una proporción considerable de perfiles pertenecientes a esta última (un 32\% respecto al 68\% de la EPC) entre quienes marcan las pautas de la conversación. De acuerdo con la clasificación de los perfiles, observamos una EPC dominada por actores políticos (49\%) y mediáticos (51\%) y una EPP configurada por ciudadanos activistas (46,4\%), activistas anónimos $(28,6 \%)$ y plataformas activistas $(10,7 \%)$. Aunque existe un pequeño porcentaje de perfiles cuyo objetivo es el humor y no la reflexión (14,3\%), debe destacarse la presencia de un alto número de ciudadanos activistas y de perfiles activistas anónimos entre quienes lideraron la discusión sobre el anuncio de la Ley del referéndum en Twitter.

En lo que se refiere a los constructores (figura 3), observamos la desproporción existente entre los principales generadores de RT, que representan 2.726 nodos, y los principales receptores (líderes de influencia), que son menos de 90 . La máxima concentración de constructores se da en la comunidad 24 (tal como sucede con los líderes de influencia) con un $47,68 \%$ que representan un $21,3 \%$ de los habitantes de toda la comunidad. En las comunidades contrarias a la celebración del referéndum, la 16 y la 17, observamos que el valor de su hub centrality es muy bajo. Esto corrobora que estas comunidades tienen un impacto menor en el debate online que aquellas que apoyan el referéndum.

Con base en la desproporción existente entre la cantidad de nodos que generan enlaces respecto a los que los reciben ${ }^{3}$ obtenemos 83 perfiles que ejercen la función de puentes de la red de RT. Para determinar si actúan realmente como puentes entre comunidades o dentro de la misma comunidad, hemos aumentado el parámetro Resolution del algo-

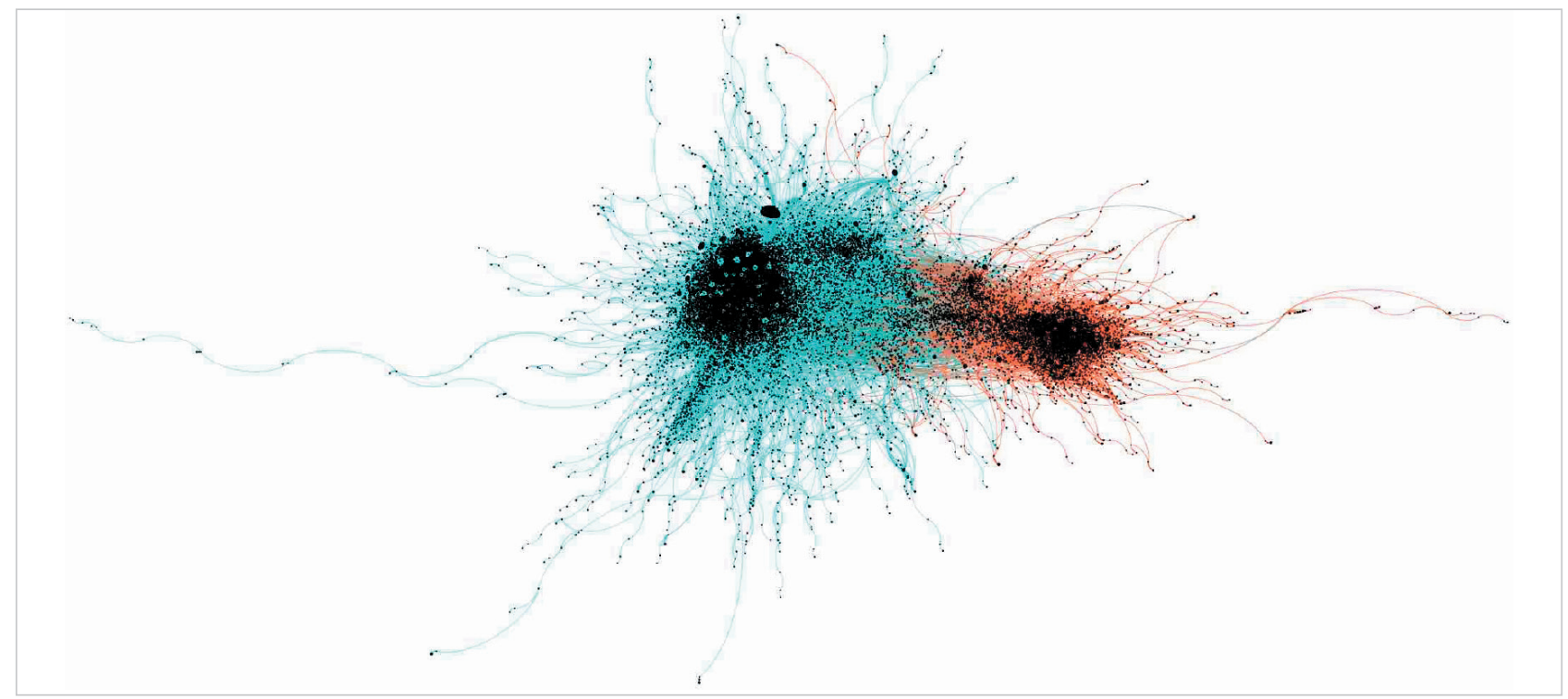

Figura 4. Mapa de la red de RT formada por la comunidad 1 y comunidad 2 
ritmo de la Modularity. Esto permite concentrar las comunidades en agrupaciones mayores hasta formar dos únicas comunidades: la comunidad 1 , posicionada a favor del referéndum, y la comunidad 2, que se define en contra (figura 4).

A partir de los parámetros obtenidos de los 83 perfiles, hemos estudiado su red de ego para confirmar si actúan a nivel local o a nivel de red global. En el caso de que haya algún nodo con aproximadamente un $50 \%$ de los habitantes de su red de ego de cada comunidad, significará que este perfil actuará como puente global, puesto que su acción unirá ambas comunidades. De no ser así, su comportamiento será de ámbito local, lo que significa que su actividad se limitará a enlazar contenidos dentro de una misma comunidad. Los resultados nos indican que todos los puentes (figura 5) actúan prácticamente a nivel local y que entre los puentes de la comunidad 1 -sí al referéndum-, que contiene un 70,23\%\% de la población total y los puentes de la comunidad $2(29,77 \%)$-no al referéndum-, las conexiones son mínimas, y viceversa, corroborando también la elevada polarización de la red a nivel de estos perfiles. Evidentemente, no podemos afirmar de manera rotunda que la polarización es total, pero sí evidenciamos que ésta existe tanto a nivel de líderes de comunidades como a nivel de perfiles puente.

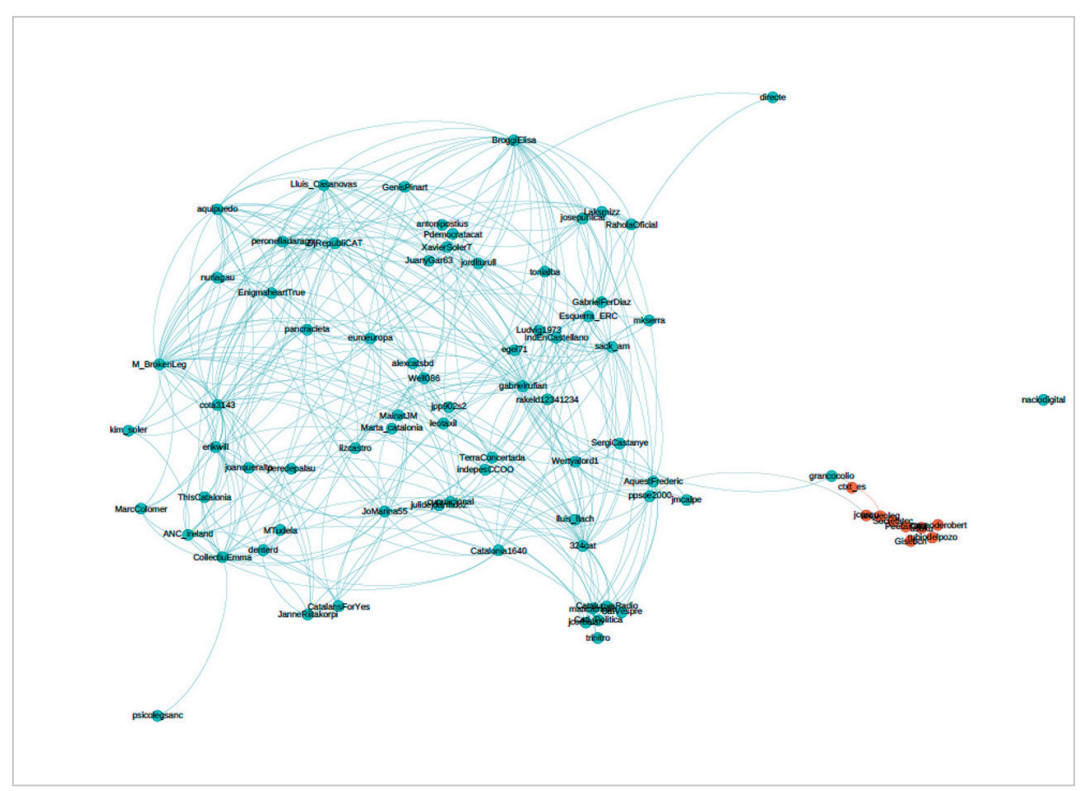

Figura 5. Mapa de Puentes en la red de RT

En el análisis cualitativo se han identificado 300 expresiones metafóricas en los 1.681 tweets analizados. Después de eliminar duplicidades -la misma expresión repetida por distintas fuentes solamente se ha codificado una vez-, se ha configurado un corpus de trabajo compuesto por 281 metáforas que se utilizaron para enmarcar la Ley del referéndum y otros target domain relativos al caso de estudio analizado (p. ej. "procés", independencia, referéndum, relación Cataluña/España, Carles Puigdemont o Mariano Rajoy). Tal como muestra la figura 6, entre los dominios que aparecen con más frecuencia en los tweets emitidos por los líderes de influencia sobresalen las expresiones relacionadas con el conFLICTO/GUERRA (35\%) y el VIAJE/CAMINO/MOVIMIENTO (27\%), seguidas de aquellas derivadas del JUEGO/DEPORTE (11\%) Y de dominios menos recurrentes como FAMILIA/AMOR/AMISTAD, ESPECTÁCULO/CINE O MECÁNICA/FÍSICA (menOS de un 4\%) Y otros (menos de un 2,5\%).

La mayor parte de las afirmaciones que contienen metáforas codificadas bajo el source domain CONFLICTO/GUERRA presentan la promulgación de la Ley del referéndum de autodeterminación por parte de los grupos parlamentarios de Junts pel Sí (JXSi) y de la Candidatura d'Unitat Popular (CUP) como un "desafío de los que nos quieren devolver al pasado" (@ PPopular); un "panfleto antidemocrático" (@rosadiezglez); un “chantaje” (@ ALevySoler vía @PPopular); o un "golpe de estado" (@CCivicaCatalana). Se trata de aquellos tweets emitidos por perfiles contrarios al movimiento de secesión y que señalan a quienes lo promueven como "golpistas" (@Jordi_Canyas, @ Er_Richal, @Santi_ABASCAL) y al actual gobierno catalán como "una dictadura comme il faut” (@societatcc). El principal actor en la vulneración de la Constitución y del estado de derecho es el presidente de la Generalitat, Carles Puigdemont, quien es tildado de "caba-

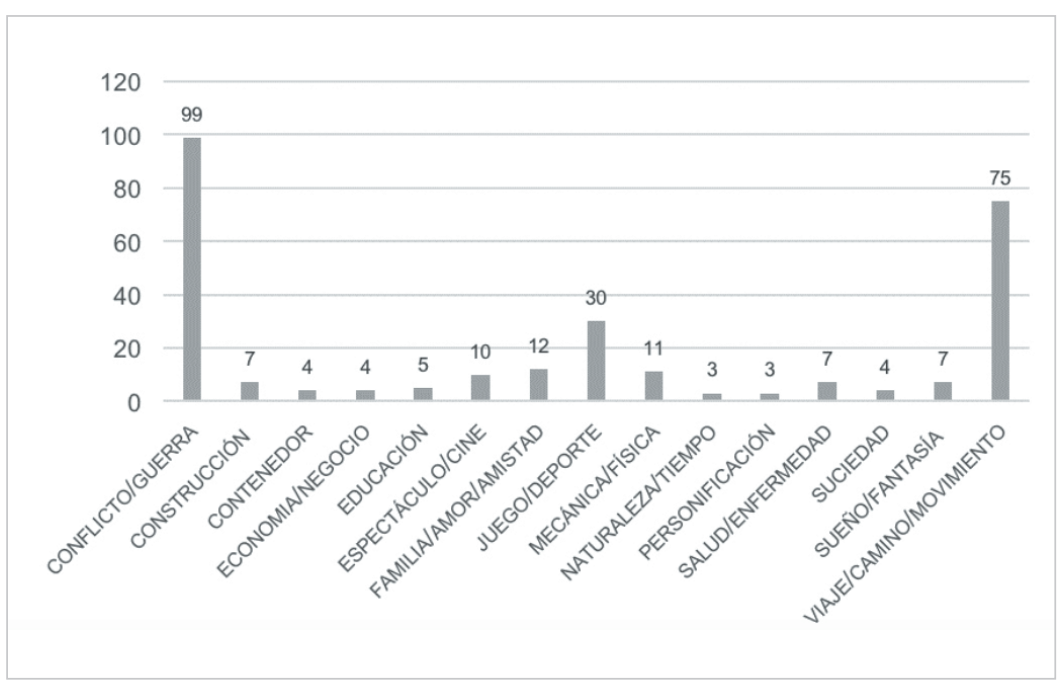

Figura 6. Source domain utilizados por los líderes de influencia 
Ilo de Troya de la CUP" (@jordi_canyas) y acusado de "neutralizar a la oposición" (@PPopular); y cuya gestión al frente del proceso independentista responde a "delirios autoritarios y frentistas" (@marianorajoy vía @PPopular).

Por otro lado, los perfiles pro-independencia conciben la relación entre Cataluña y España como "una batalla desigual" (@leotaxil). En esta batalla, se sigue delegando en los catalanes la responsabilidad de "combatir contra la España de la transición" (@leotaxil); un “ejército de demócratas" (@jcuixart) que, con su actuación, debe "liberar" Cataluña, "sometida a un Estado fascista y agresor" (@leotaxil). Usuarios como @eduardcabus hacen un llamamiento a "independizar[se] en defensa propia" y @ForcadellCarme manifiesta que "estamos amenazados, pero no nos rendiremos". Contrariamente al posicionamiento de los perfiles pertenecientes a la comunidad 2, para la comunidad 1 la Ley del referéndum no es un golpe de estado, sino que se define como la "respuesta democrática a la ruptura del pacto constitucional del 78" (@martarovira vía@naciopolitica).

Hablar sobre el proceso independentista en términos de VIAJE/CAMINO/MOVIMIENTO se ha convertido en la forma dominante de enmarcar el discurso político y mediático en los últimos años. Lo que para unos es "un globo pinchado que en su caída va golpeando al Parlament y las leyes" (Pablo Casado vía @okdiario), para otros es un "camino" que "no se puede parar", donde la Ley del referéndum vehicula "el final del recorrido" (@JuntspelSí). Aunque en esta ocasión, las posturas opuestas entre la Generalitat de Catalunya y el Gobierno de España no llevan a un "choque de trenes", sino a una nueva situación dónde "un tren continuará y el [tren] del statu quo permanecerá" (@KRLS). Esta sensación de "avanzar” (@eduardvoltas); “empezar un camino” (@lluis_Ilach vía @JuntspelSí); “dar un paso más” (@yeyaboya); “tirar millas" (@sumate_asoc) o “[dar] gas a fondo” (@vpartal), contrasta con la Cataluña “paralizada” (@PPopular) y “atascada" (@Societatcc).

Para la comunidad 1, el 1 de octubre actúa como el hilo de Ariadna, que permitirá a Cataluña salir del laberinto en el que se encuentra, mientras que la comunidad 2 lo considera un "freno" (@okdiario) para el desarrollo económico de la región. Cabe destacar que existen visiones con un tono más conciliador que establecen que "nunca es tarde si se evita una huida hacia un precipicio" mientras señala que deben hacerse "las reformas necesarias para continuar caminando juntos" (@patxilopez vía @PSOE), eso es, “dar un paso y una salida política” (@sanchezcastejon) al conflicto.

Las metáforas de JUEGO/DEPORTE se usan de forma exclusiva en la comunidad 1 para presentar la convocatoria del referéndum como una competición deportiva. Se habla de la sindicatura electoral como "los árbitros del referéndum" (@Arapolitica) al tiempo que se afirma que los catalanes “no nos pensamos tragar ningún gol” (@elgatnegre007). El juego de ajedrez -“estamos muy cerca del jaque mate a Españistán” (@egel71) o “el tablero de juego es, como mínimo, europeo” (@VilaWeb)- es también un recurso utilizado por los actores pro-independencia.

Otras metáforas utilizadas para definir la Ley del referéndum o bien la relación entre España y Cataluña son aquellas relacionadas con la FAMILIA/AMOR/AMISTAD ("Violar la Constitución” @jordi_canyas; “separarse con España" @reuters); ESPECTÁculo/CINE ("performance" C's vía @324cat para “entretener al personal" Sáenz de Santamaría vía @324cat, "Si quieres teatro o circo,

Entre los dominios que aparecen con más frecuencia en los tweets emitidos por los influencers sobresalen las expresiones relacionadas con el conflicto/guerra (35\%) y el viaje/camino/movimiento (27\%)

te pagas la entrada” @rosadiezglez); o MECÁNICA/FísICA (“romper con España” @patxilopez vía @PSOE, “romper la convivencia en Cataluña" González, Aznar y Zapatero vía @elconfidencial, “mecanismos para poner las urnas" @ naciodigital).

\section{Discusión y conclusiones}

La pregunta inicial de esta investigación plantea cuáles son las características del debate online con el objetivo de discernir si Twitter puede considerarse una red que contribuye a la discusión horizontal entre políticos, medios y ciudadanos o si por el contario sigue perpetuando la hegemonía de los dos primeros en la creación de la opinión pública (Mazzoleni, 2010). En este sentido, la identificación estadística de los líderes de influencia en el debate sobre el anuncio de la Ley del referéndum permite afirmar que si bien los perfiles asociados a la EPC (esfera pública central), un 68\%, continúan ejerciendo un papel relevante en la discusión, los usuarios que forman parte de la sociedad civil (32\%) también tienen algo que decir. Puede sostenerse que en el estudio que nos ocupa, las EPP (esferas públicas periféricas) aprovecharon la horizontalidad que les brindaba Twitter para situar sus puntos de vista en el centro del debate; y lo que es más importante, fueron escuchados por otros usuarios. Del mismo modo, el hecho de que exista un porcentaje tan elevado de perfiles pertenecientes a la esfera pública central en una red concebida para el intercambio de opiniones entre la ciudadanía, corrobora las tesis de Jenkins (2006) en relación con la lógica estratégica de los poderes tradicionales. Desde el momento en el que Twitter es considerado un nido de ciudadanos prescriptores en cuestiones políticas (Rodríguez-Andrés; Ureña-Uceda, 2011), dichos poderes perciben su participación en los medios sociales como

La interacción entre participantes existe, pero se limita a quienes comparten posicionamientos similares sobre el conflicto analizado 
una táctica más dentro de su estrategia de comunicación. Si utilizan las posibilidades de interacción con los usuarios y si contribuyen así a una mejora de la calidad democrática, es una cuestión que escapa a esta investigación.

Por otro lado, la figura 4 detalla cómo la mayoría de perfiles que ejercen de puentes entre usuarios actúan de forma local, es decir, se comunican sólo dentro de su comunidad. La interacción entre participantes existe, pero los datos indican que se limita a quienes comparten posicionamientos similares sobre el conflicto analizado. La práctica inexistencia de puentes globales demuestra que la discusión, en este caso, está polarizada. Esto no significa que no existan vínculos entre comunidades, pero éstos no se dan en las que contienen nodos con mayor relevancia en términos de enlaces recibidos.

Si nos centramos en los resultados obtenidos con el análisis de la muestra (n2), vemos que la polarización que se percibe a nivel estadístico se traslada también al ámbito discursivo. Mediante la detección de las metáforas empleadas de forma más frecuente por parte de los líderes de influencia, hemos identificado los dominios con que los perfiles pertenecientes a la comunidad 1 y a la comunidad 2 enmarcan el asunto discutido. Dado el contexto que rodeó el anuncio de la Ley del referéndum y, apoyándonos en la división gráfica entre comunidades, no es extraño que el source domain utilizado con más frecuencia por parte de ambas fuera el de CONFLICTO/ GUERRA. En este sentido, recurrimos a la noción de scenario (Musolff, 2004) para agrupar las expresiones por comunidades e identificar cuál es la narrativa que desarrolla los mapas conceptuales. El scenario articula las diferentes narrativas o perspectivas posibles de un source domain en contexto y por ello guía la interpretación del asunto político conceptualizado a través del uso de la metáfora. Así, aunque bajo un mismo marco de conflicto, los resultados

Los resultados evidencian dos narrativas diametralmente opuestas: por un lado, el anuncio de la Ley del referéndum es un golpe de estado (comunidad 2) y por el otro es garantía de libertad y democracia (comunidad 1) evidencian dos narrativas diametralmente opuestas. Por un lado, el anuncio de la Ley del referéndum es un golpe de estado (comunidad 2) y, por el otro, el anuncio de la Ley del referéndum es garantía de libertad y democracia (comunidad 1). El hecho de que los perfiles integrados en las dos comunidades usen mayoritariamente un léxico bélico para referir la situación política en Cataluña, refuerza la concepción de los participantes en el conflicto como dos bandos opuestos y enfrentados y dificulta la elaboración de una narrativa alternativa.

Las reacciones que se generan en Twitter en forma de red de RT (retweets) alrededor de un determinado hecho son un reflejo de lo que sucede en la vida real debido a este hecho. Las comunidades formadas responden a las agrupaciones por afinidad existentes ya en la sociedad; los partidarios y detractores del referéndum retroalimentan sus propias creencias y argumentarios posibilitando la creación de opinión pública a nivel local, pero no así en el global. Podríamos pensar entonces que Twitter puede potenciar el aislamiento y la construcción de comunidades que funcionan con la lógica de las cámaras de eco. Sin embargo, pensamos que el entorno es la causa. Es decir, la morfología de la red es consecuencia del posicionamiento ideológico de los nodos participantes que la conforman. Así, se constata que, aunque las redes sociales amplían el espacio de la influencia a nuevos actores, el liderazgo de las EPP en la conversación no es sinónimo de deliberación sobre asuntos públicos entre las comunidades que lideran y que la red se configura a partir de clústeres de perfiles que comparten una visión similar sobre el asunto público discutido. Estas afirmaciones son válidas para el presente caso de estudio. Futuras investigaciones deberían complementar el análisis de la red de RT con la red de menciones con el fin de comprobar si dicha situación se reproduce también allí y verificar hasta qué punto las redes de RT y de mención que se generan alrededor de casos políticos conflictivos reproducen un patrón similar o bien presentan diferencias.

Esta limitación responde a la idiosincrasia de este trabajo, que se plantea como un estudio exploratorio que propone la triangulación metodológica como aproximación para abordar el análisis de redes. Los investigadores son conscientes de otras limitaciones como las dificultades que plantea Twitter para la obtención de la muestra completa. Así, aunque se ha trabajado con una muestra cuantitativa que contenía el mayor número de tweets posible, es necesario remarcar que la red social no nos brinda la totalidad de los perfiles que participaron de la conversación. En términos de morfología de la red, sería interesante identificar qué porcentaje de tweets son retuiteados dentro de su propia comunidad y qué porcentaje la transciende y llega a otras comunidades, puesto que ello nos proporcionaría más datos acerca de la polarización, que complementarían el análisis de los puentes llevado a cabo en este trabajo.

\section{Notas}

1. En el período temporal analizado Twitter todavía no había ampliado el espacio para escribir un tweet a 280 caracteres.

2. Los nodos suman un $95,4 \%$, puesto que el porcentaje restante se refiere a comunidades residuales.

3. Los 155.396 enlaces que conforman la red han sido generados por 3.918 nodos con un valor mínimo de 1 enlace/nodo y un valor máximo de 495 enlaces/nodo. Los enlaces recibidos, en cambio, quedan concentrados en 5.901 nodos cuyo valor mínimo es 1 y el máximo es 7.559. Viendo esta desproporción -la primera está muy distribuida, mientras que la segunda está concentrada en pocos nodos- decidimos fijar el umbral del valor menor de WI (Weighted Indegree) de los nodos que concentren el $75 \%$ de los enlaces recibidos (116.547) y el umbral de WO (Weighted Outdegree) será el valor menor de los nodos que generen el $50 \%$ de los enlaces (77.698). Obtenemos 350 perfiles que cumplen con el umbral del WI y 2.726 que lo hacen con el WO. Del cruce de estos perfiles determinamos los 83 puentes mencionados. 


\section{Referencias}

Alvídrez, Salvador; Franco-Rodríguez, Oziel (2016). “Estilo comunicativo súbito en Twitter: efectos sobre la credibilidad y la participación cívica". Comunicar, v. 24, n. 47, pp. 89-97.

https://doi.org/10.3916/C47-2016-09

Aragón, Pablo; Kappler, Karolyn-Eva; Kaltenbrunner, Andreas; Laniado, David; Volkovich, Yana (2013). “Communication dynamics in Twitter during political campaigns: The case of the 2011 Spanish national election". Policy \& internet, v. 5, n. 2, pp. 183-206.

https://doi.org/10.1002/1944-2866.POI327

Ausserhofer, Julian; Maireder, Axel (2013). "National politics on Twitter. Structures and topics of a networked public sphere". Information, communication \& society, v. 16, n. 3, pp. 291-314.

https://doi.org/10.1080/1369118X.2012.756050

Bakshy, Eytan; Hofman, Jake M.; Mason, Winter A.; Watts, Duncan J. (2011). "Everyone's an influencer: Quantifiyng influence on Twitter". In: Proceedings of the Forth ACM Intl conf on web search and web data mining, pp. 65-74. https://doi.org/10.1145/1935826.1935845

Barassi, Veronica; Treré, Emiliano (2012). “Does web 3.0 come after web 2.0? Deconstructing theoretical assumptions through practice". New media \& society, v. 14, n. 8, pp. 1269-1285.

https://doi.org/10.1177/1461444812445878

Barberá, Pablo; Jost, John T.; Nagler, Jonathan; Tucker, Joshua A.; Bonneau, Richard (2015). "Tweeting from left to right: Is online political communication more than an echo chamber?". Psychological science, v. 26, n. 10, pp. 1531-1542. https://doi.org/10.1177/0956797615594620

Barnidge, Matthew (2017). “Exposure to political disagreement in social media versus face-to-face and anonymous online settings". Political communication, v. 34, n. 2, pp. 302-321.

https://doi.org/10.1080/10584609.2016.1235639

Boutet, Antoine; Kim, Hyoungshick; Yoneki, Eiko (2013). "What's in Twitter: I know what parties are popular and who you are supporting now!". Social network analysis and mining, v. 3, n. 4, pp. 1379-1391.

https://doi.org/10.1007/s13278-013-0120-1

Boutyline, Andrei; Willer, Robb (2017) "The social structure of political echo chambers: Variation in ideological homophily in online networks". Political psychology, v. 38, n. 3, pp. 551-569.

https://doi.org/10.1111/pops.12337

Bruns, Axel; Highfield, Tim (2015). "Is Habermas on Twitter? Social media and the public sphere". In: Bruns, Axel; Enli, Gunn; Skogerbø, Eli; Larsson, Anders-Olof; Christensen, Christian (eds.). The Routledge companion to social media and politics. New York: Routledge, pp. 56-73. ISBN: 9781138860766

https://eprints.qut.edu.au/91810

Burgers, Christian; Konijn, Elly A.; Steen, Gerard J. (2016). "Figurative framing: Shaping public discourse through metaphor, hyperbole, and irony". Communication theory, v. 26, n. 4, pp. 410-430.

https://doi.org/10.1111/comt.12096

Casero-Ripollés, Andreu (2018). "Research on political information and social media: Key points and challenges for the future". El profesional de la información, v. 27, n. 5, pp. 964-974.

https://doi.org/10.3145/epi.2018.sep.01

Cha, Meeyoung; Haddadi, Hamed; Benevenuto, Fabrício; Gummadi, Krishna P. (2010). "Measuring user influence in Twitter: The million follower fallacy". In: Proceedings of the Fourth Intl AAAI Conf on weblogs and social media, pp. 10-17. http://twitter.mpi-sws.org/icwsm2010_fallacy.pdf

Charteris-Black, Jonathan (2004). Corpus approaches to critical metaphor analysis. Basingstoke, Hampshire: Palgrave Macmillan. ISBN: 9781403932921

Charteris-Black, Jonathan (2011). Politicians and rhetoric. The persuasive power of metaphor. Basingstoke, Hampshire: Palgrave Macmillan. ISBN: 9780230251649

Cherven, Ken (2015). Mastering Gephi network visualization. Birmingham: Packt Publishing. ISBN: 9781783987344

Choi, Sujin (2015). "The two-step flow of communication in Twitter-based public forums". Social science computer review, v. 33, n. 6, pp. 696-711.

https://doi.org/10.1177/0894439314556599

Colleoni, Elanor; Rozza, Alessandro; Arvidsson, Adam (2014). “Echo chamber or public sphere? Predicting political orientation and measuring political homophily in Twitter using big data". Journal of communication, v. 64, n. 2, pp. $317-332$.

https://doi.org/10.1111/jcom.12084 
Conover, Michael D.; Ratkiewicz, Jacob; Francisco, Matthew R.; Gonçalves, Bruno; Flammini, Alessandro; Menczer, Filippo (2011). "Political polarization on Twitter". In: Proceedings of the Fifth Intl AAAI conf on weblogs and social media, pp. 89-96.

https://www.researchgate.net/publication/221297916_Political_Polarization_on_Twitter

Crameri, Kathryn (2015). "Political power and civil counterpower: The complex dynamics of the Catalan independence movement". Nationalism and ethnic politics, v. 21, n. 1, pp. 104-120.

https://doi.org/10.1080/13537113.2015.1003491

D’heer, Evelien; Verdegem, Pieter (2014). "Conversations about the elections on Twitter: Towards a structural understanding of Twitter's relation with the political and the media field". European journal of communication, v. 29, n. 6, pp. 720-734.

https://doi.org/10.1177/0267323114544866

Dahlgren, Peter (2005). "The internet, public spheres, and political communication: Dispersion and deliberation". Political communication, v. 22, n. 2, pp. 147-162.

https://doi.org/10.1080/10584600590933160

Dang-Xuan, Linh; Stieglitz, Stefan; Wladarsch, Jennifer; Neuberger, Christoph (2013). “An investigation of influentials and the role of sentiment on political communication on Twitter during election periods". Information, communication \& society, v. 16, n. 5, pp. 795-825.

https://doi.org/10.1080/1369118X.2013.783608

Dubois, Elizabeth; Blank, Grant (2018). "The echo chamber is overstated: The moderating effect of political interest and diverse media. Information, communication \& society, v. 21, n. 5, pp. 729-745.

https://doi.org/10.1080/1369118X.2018.1428656

Dubois, Elizabeth; Gaffney, Devin (2014). "The multiple facets of influence: Identifying political influentials and opinion leaders on Twitter". American behavioral scientist, v. 58, n. 10, pp. 1260-1277.

https://doi.org/10.1177/0002764214527088

Esteve-Del-Valle, Marc; Borge-Bravo, Rosa (2018). "Eco chambers in parliamentary Twitter networks: The Catalan case". International journal of communication, v. 12, pp. 1715-1735.

https://ijoc.org/index.php/ijoc/article/view/8406

Feller, Albert; Kuhnert, Matthias; Sprenger, Timm O.; Welpe, Isabell M. (2011). “Divided they tweet: The network structure of political microbloggers and discussion topics". In: Proceedings of the Fifth Intl AAAl Conf on weblogs and social media, pp. 474-477.

https://pdfs.semanticscholar.org/1f6d/ac76be7d0415d9836adfb8ce14ab484d7229.pdf

Feng, Yang (2016). "Are you connected? Evaluating information cascades in online discussion about the \#RaceTogether campaign". Computers in human behavior, v. 54, pp. 43-53.

https://doi.org/10.1016/j.chb.2015.07.052

Flaxman, Seth; Goel, Sharad; Rao, Justin M. (2016). "Filter bubbles, echo chambers, and online news consumption". Public opinion quarterly, v. 80, n. S1, pp. 298-320.

https://doi.org/10.1093/poq/nfw006

Gamson, William-Anthony; Modigliani, Andre (1989). "Media discourse and public opinion on nuclear power: A constructionist approach". American journal of sociology, v. 95, n. 1, pp. 1-37.

https://doi.org/10.1086/229213

Gil de Zúñiga, Homero; Molyneux, Logan; Zheng, Pei (2014). “Social media, political expression, and political participation: Panel analysis of lagged and concurrent relationships". Journal of communication, v. 64, n. 1, pp. 612-634.

https://doi.org/10.1111/jcom.12103

González-Bailón, Sandra; Borge-Holthoefer, Javier; Moreno, Yamir (2013). "Broadcasters and hidden influentials in online protest diffusion". American behavioral scientist, v. 57, n. 7, pp. 943-965.

https://doi.org/10.1177/0002764213479371

González-Bailón, Sandra; Wang, Ning (2016). "Networked discontent: The anatomy of protest campaigns”. Social networks, v. 44, pp. 95-104.'

https://doi.org/10.1016/j.socnet.2015.07.003

Grau-Masot, Josep-Maria (2018). “Les xarxes d’influència i de construcció d’opinió pública a Twitter. El cas del procés d'independència de Catalunya: Des de I'anunci de la Llei del Referèndum fins als fets d'octubre". Tesis doctoral. Universitat Rovira i Virgili, Tarragona.

https://www.tesisenred.net/handle/10803/664742 
Greimas, Algirdas-Julius (1987). “La isotopía del discurso". En: Greimas, Algirdas-Julius. Semántica estructural: investigación metodológica. Madrid: Gredos, pp. 105-155. ISBN: 9788424911799

Gruzd, Anatoliy (2012). "Investigating political polarization on Twitter: A Canadian perspective". In: Internet, politics, policy: Big data, big challenges?, pp. 1-11.

https://socialmedialab.ca/2012/09/20/investigating-political-polarization-on-twitter-a-canadian-perspective

Guerrero-Solé, Frederic (2017). “Community detection in political discussions on Twitter: An application of the retweet overlap network method to the Catalan process toward independence". Social science computer review, v. 35, n. 2, pp. $244-261$. https://doi.org/10.1177/0894439315617254

Guerrero-Solé, Frederic; Corominas-Murtra, Bernat; López-González, Hibai (2014). “Pacts with Twitter. Predicting voters' indecision and preferences for coalitions in multiparty systems". Information, communication \& society, v. 17, n. 10, pp. $1280-1297$.

https://doi.org/10.1080/1369118X.2014.920040

Guibernau, Montserrat (2013). "Secessionism in Catalonia: After democracy". Ethnopolitics, v. 12, n. 4, pp. 368-393. https://doi.org/10.1080/17449057.2013.843245

Hilbert, Martin; Vásquez, Javier; Halpern, Daniel; Valenzuela, Sebastián; Arriagada, Eduardo (2016). “One step, two step, network step? Complementary perspectives on communication flows in twittered citizen protests". Social science computer review, v. 35, n. 4, pp. 444-461.

https://doi.org/10.1177/0894439316639561

Holsti, Ole R. (1969). Content analysis for the social sciences and humanities. Reading, MA: Addison-Wesley. ISBN: 978 0201029406

Hsu, Chien-Leng; Park, Han-Woo (2012). "Mapping online social networks of Korean polititians". Government information quarterly, v. 29, n. 2, pp. 169-181.

https://doi.org/10.1016/j.giq.2011.09.009

Jenkins, Henry (2006). Convergence culture: Where old and new media collide. New York: New York University Press. ISBN: 9780814742952

Jungherr, Andreas; Jürgens, Pascal (2014). "Through a glass, darkly. Tactical support and symbolic association in Twitter messages commenting on Stuttgart 21". Social science computer review, v. 32, n. 1, pp. 74-89.

https://doi.org/10.1177/0894439313500022

Katz, Elihu; Lazarsfeld, Paul F. (1955). Personal influence. The part played by people in the flow of mass communications. New York: Routdlege. ISBN: 9781351500197

Klinger, Ulrike; Svensson, Jakob (2015). "The emergence of network media logic in political communication: A theoretical approach". New media \& society, v. 17, n. 8, pp. 1241-1257.

https://doi.org/10.1177/1461444814522952

Kövecses, Zoltan (2010). Metaphor: a practical introduction. Oxford: Oxford University Press. ISBN: 9780199888429

Larsson, Anders-Olof; Moe, Hallvard (2011). "Studying political microblogging: Twitter users in the 2010 Swedish election campaign". New media \& society, v. 14, n. 5, pp. 729-747.

https://doi.org/10.1177/1461444811422894

Loader, Brian D.; Mercea, Dan (2011). "Networking democracy? Social media innovations and participatory politics". Information, communication \& society, v. 14, n. 6, pp. 757-769.

https://doi.org/10.1080/1369118X.2011.592648

López-García, Guillermo (2006). “Comunicación en red y mutaciones de la esfera pública”. Zer, v. 11, n. 20, pp. $231-249$. https://www.ehu.eus/ojs/index.php/Zer/article/view/3762

Margolis, Michael; Resnick, David (2000). Politics as usual: The cyberspace 'revolution'. London: Sage Publications. ISBN: 9780761913313

https://doi.org/10.4135/9781452233475

Masip, Pere; Guallar, Javier; Suau, Jaume; Ruiz-Caballero, Carlos; Peralta, Miquel (2015). “News and social networks: audience behavior". El profesional de la información, v. 24, n. 4, pp. 363-370.

https://doi.org/10.3145/epi.2015.jul.02

Mazzoleni, Gianpietro (2010). La comunicación política. Madrid: Alianza Editorial. ISBN: 9788420669403

McNair, Brian (2007). An introduction to political communication. London: Routledge. ISBN: 9780415596442

McPherson, Miller; Smith-Lovin, Lynn; Cook, James M. (2001). "Birds of a feather: Homophily in social networks". Annual review of sociology, v. 27, pp. 415-444. 
https://doi.org/10.1146/annurev.soc.27.1.415

Moragas-Fernández, Carlota M.; Montagut, Marta; Capdevila, Arantxa (2018). "The process en route: the metaphor of the journey as the dominant narrative for the political discourse in Catalonia". Critical discourse studies, v. 15, n. 5, pp. 517-539. https://doi.org/10.1080/17405904.2018.1468787

Musolff, Andreas (2004). Metaphor and political discourse. Analogical reasoning in debates about Europe. Basingstoke, Hampshire: Palgrave Macmillan. ISBN: 9781403933898

Musolff, Andreas (2006). "Metaphor scenarios in public discourse". Metaphor and symbol, v. 21, n. 1, pp. 23-38. https://doi.org/10.1207/s15327868ms2101_2

Musolff, Andreas (2012). "The study of metaphor as part of critical discourse analysis". Critical discourse studies, v. 9, n. 3, pp. 301-310.

https://doi.org/10.1080/17405904.2012.688300

Newman, Mark (2010). Networks: An introduction. Oxford: Oxford University Press. ISBN: 9780199206650

Rodríguez-Andrés, Roberto; Ureña-Uceda, Daniel (2011). “Diez razones para el uso de Twitter como herramienta en la comunicación política y electoral". Comunicación y pluralismo, n. 10, pp. 89-116.

http://dadun.unav.edu/handle/10171/35625

Sampedro, Víctor (2000). Opinión pública y democracia deliberativa. Medios, sondeos y urnas. Madrid: Ediciones Istmo. ISBN: 9788470903823

Sampedro, Víctor (2011). "Introduction: New trends and challenges in political communication". International journal of press/politics, v. 16, n. 4, pp. 431-439.

https://doi.org/10.1177/1940161211418291

Sampedro, Víctor; Resina-De-la-Fuente, Jorge (2010). "Opinión pública y democracia deliberativa en la sociedad red”. Ayer. Revista de historia contemporánea, v. 80, pp. 139-162.

Scott, John (2013). Social network analysis. London: Sage Publications. ISBN: 9781446209042

Scott, John; Carrington, Peter J. (2012). The SAGE handbook of social network analysis. London: Sage Publications. ISBN: 9781847873958

Semino, Elena (2008). Metaphor in discourse. Cambridge: Cambridge University Press. ISBN: 9780521686969

Serrano, Ivan (2013). "Just a matter of identity? Support for independence in Catalonia". Regional \& federal studies, v. 23, n. 5, pp. 523-545.

https://doi.org/10.1080/13597566.2013.775945

Sunstein, Cass R. (2003). Why societies need dissent. Cambridge, MA: Harvard University Press. ISBN: 9780674017689

Sunstein, Cass R. (2008). "Neither Hayek nor Habermas". Public choice, v. 134, n. 1/2, pp. 87-95.

https://doi.org/10.1007/s11127-007-9202-9

Sup-Park, Chang (2013). “Does Twitter motivate involvement in politics? Tweeting, opinion leadership, and political engagement". Computers in human behavior, v. 29, n. 4, pp. 1641-1648.

https://doi.org/10.1016/j.chb.2013.01.044

Vaccari, Cristian; Valeriani, Augusto; Barberá, Pablo; Jost, John T.; Nagler, Jonathan; Tucker, Joshua A. (2016). “Of echo chambers and contrarian clubs: Exposure to political disagreement among German and Italian users of Twitter". Social media + society, v. 2, n. 3, pp. 1-16.

https://doi.org/10.1177/2056305116664221

Veenstra, Aaron S.; Narayanan, Iyer; Sup-Park, Chang; Alajmi, Fawaz (2015). “Twitter as 'a journalistic substitute'? Examining \#wiunion tweeters' behavior and self-perception". Journalism, v. 16, n. 4, pp. 488-504.

https://doi.org/10.1177/1464884914521580

Witschge, Tamara; Anderson, Christopher W.; Domingo, David; Hermida, Alfred (2016). The SAGE handbook of digital journalism. London: Sage Publications. ISBN: 9781473906532

Yang, Shuzhe; Quan-Haase, Anabel; Rannenberg, Kai (2016). “The changing public sphere on Twitter: Network structure elites and topics of the \#righttobeforgotten". New media \& society, v. 19, n. 12, pp. 1983-2002.

https://doi.org/10.1177/1461444816651409

Yardi, Sarah; Boyd, Danah (2010). "Dynamic debates: An analysis of group polarization over time on Twitter". Bulletin of science, technology \& society, v. 30, n. 5, pp. 316-327.

https://doi.org/10.1177/0270467610380011 